\title{
II rapporto impresa-territorio tra efficienza locale, efficacia di contesto e sostenibilità ambientale
}

\author{
Sergio Barile ${ }^{*}$ Marialuisa SaVIANo** \\ Francesco POLESE*** PrIMIANO DI NAUTA ${ }^{* * *}$
}

\begin{abstract}
Obiettivo del paper: Il paper propone un modello di rappresentazione del territorio come sistema dinamico di relazioni intersoggettive capace di garantire non solo l'efficienza dei processi interni all'organizzazione sviluppata, ma anche risultati efficaci nel contesto complessivo e un impatto sostenibile nell'ambiente più esteso.

Metodologia: Il contributo è sviluppato sulla base dell'Approccio Sistemico Vitale (ASV) sia come metodologia di indagine dei fenomeni sociali sia come metodologia di governo delle organizzazioni.

Risultati: Attraverso l'impiego di concetti quali 'rilevanza', 'consonanza' e 'risonanza', l'approccio proposto conduce alla rappresentazione di un sistema territoriale vitale, capace di sopravvivere nel proprio contesto creando valore per $i$ sovrasistemi di riferimento $e$ determinando le condizioni per un equilibrio sostenibile.

Limiti della ricerca: Il paper offre un framework teorico-concettuale che potrà essere oggetto di studio e di ulteriori sviluppi anche attraverso l'analisi di casi reali e verifiche empiriche.

Implicazioni pratiche: Lo schema generale proposto è adottabile, congiuntamente ai modelli manageriali consolidati, tanto dall'attore istituzionale, che vede il territorio nel suo complesso come sistema vitale, quanto dall'attore imprenditoriale, che vede nel territorio un fertile contesto di azione dal quale trarre e al quale offrire opportunità di sviluppo in un intreccio armonico di finalità e in un'ottica condivisa di sostenibilità.

Originalità del lavoro: L'originalità del paper risiede nel proporre un modello di sintesi per uno sviluppo sinergico del territorio che catalizzi istanze aziendali, di contesto e ambientali, promuovendo la sostenibilità nei rapporti impresa-territorio.
\end{abstract}

Parole chiave: rapporto impresa-territorio; approccio sistemico vitale; efficienza; efficacia; sostenibilità

* Ordinario di Economia e Gestione delle Imprese - Sapienza Università di Roma e-mail: sergio.barile@uniroma1.it

** Associato di Economia e Gestione delle Imprese - Università degli Studi di Salerno e-mail: msaviano@unisa.it

*** Associato di Economia e Gestione delle Imprese - Università degli Studi di Salerno e-mail: fpolese@unisa.it

**** Ricercatore di Economia e Gestione delle Imprese - Università degli Studi di Foggia e-mail: p.dinauta@unifg.it 
Purpose of the paper: This paper proposes a model of territory as a dynamic system of inter-subjective relationships able to ensure not only the efficiency of processes within the organization developed, but also effective results in the overall context, and a sustainable impact in the most extended one.

Methodology: The contribution is developed on the basis of the Viable Systems Approach (VSA), both as a methodology of investigation of social phenomena and as a methodology for the governance of organizations.

Findings: By making use of concepts such as 'relevance', 'consonance' and 'resonance', the proposed approach outlines a model for the representation of a territory as viable system, able of surviving in the context creating value for supra-systems and determining the conditions for a sustainable balance.

Research limits: The paper provides a theoretical-conceptual framework that could be the starting point for a cases study, opening up opportunities for further analysis and empirical research.

Practical implications: The proposed general scheme is adoptable, in conjunction with the consolidated management models, both by the institutional actor that views the whole territory as a viable system, and by the entrepreneur that views the territory as a fertile environment from which to get and offer development opportunities in a harmonic mix of finalities and in a shared perspective of sustainability.

Originality of the study: The originality of the paper lies in proposing a synthesis model for synergistic development of a territory that catalyses contextual and environmental organizations expectations, promoting sustainability in the firm-territory relationship.

Key words: firm-territory relationship; viable systems approach; efficiency; effectiveness; sustainability

\section{Introduzione}

Il rapporto impresa-territorio si snoda in un articolato insieme di strutture da cui si sviluppano ricorsivamente le dinamiche vitali di individui, famiglie e organizzazioni nei molteplici contesti dell'economia, dell'ambiente e della società. La formazione stessa del territorio è il risultato di un dinamico evolvere di tali interazioni ${ }^{1}$, che si sviluppano nell'ambito di un definito sistema di vincoli e regole volti a disciplinare l'attività sociale ed economica nel e del territorio. Nell'ambito di tale quadro normativo, l'azione degli individui e delle organizzazioni si svolge, comunque, entro significativi gradi di libertà, che sono espressione di un'interpretazione soggettiva dell'assetto normativo codificato (codici, testi unici, ecc.). Tale interpretazione soggettiva, influenzata dal sistema di valori prevalente (categorie valoriali, morale, etica) e dai paradigmi dominanti, traduce la norma in regola comportamentale attraverso la definizione di procedure, modelli, protocolli e tecniche operative di uso comune. La regola comportamentale, quindi, risultando da un'interpretazione soggettiva della norma, nell'ambito di comunità omogenee,

1 Molti autori riconoscono la necessità di una visione dinamica del territorio. Si veda tra gli altri Painter (2006). 
assume profili sempre nuovi e sempre più distanti dal minimo comun denominatore che nel tempo è venuto a consolidarsi, dando luogo ad un dinamico evolvere di usi, costumi e consuetudini (Barile, 2009b). In sostanza, come illustrato in Fig. 1, si sviluppa un circuito in cui il singolo individuo, nell'interpretare una legge, a sua volta interpretata in una norma che ha indotto una regola, stabilisce delle consuetudini, le quali, a loro volta, influenzando la definizione delle leggi, attivano un nuovo ciclo normativo. In tale prospettiva, evidentemente, la convinzione che il quadro normativo possa influenzare in maniera deterministica il comportamento degli attori sociali non trova riscontro.

Fig. 1: La dinamica normativa tra legge, norma e regola

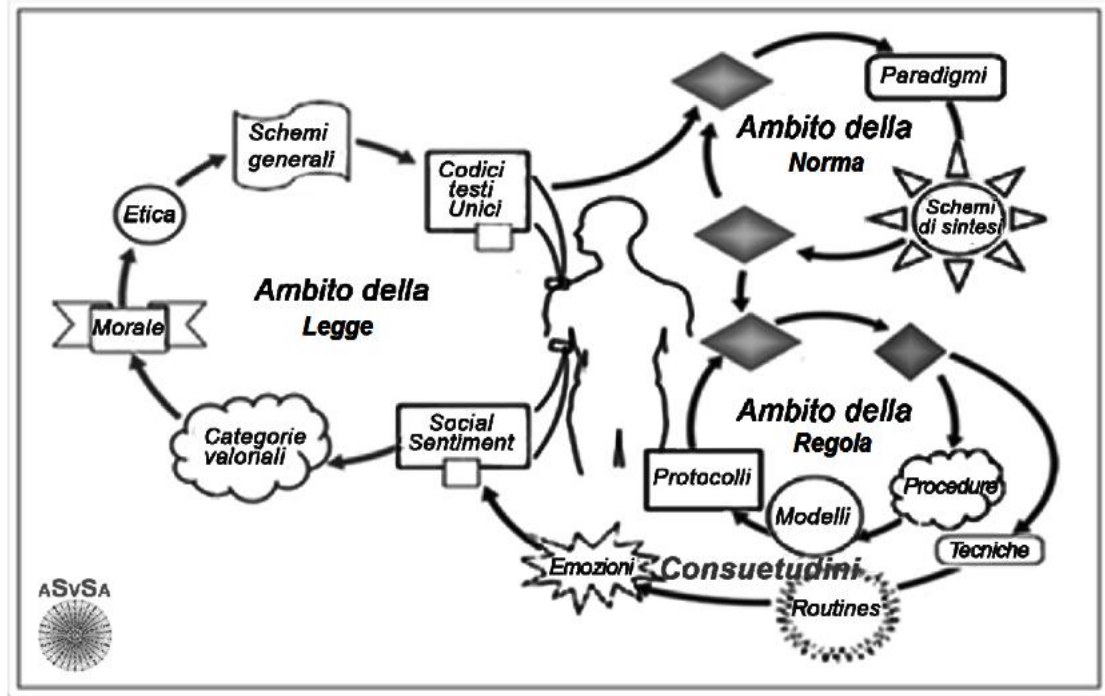

Fonte: Barile, 2011, www.asvsa.org.

Figura utilizzata con il permesso di ASvSA - Associazione per la ricerca sui Sistemi Vitali

Quanto così illustrato evidenzia come la dinamica comportale dei diversi attori coinvolti in un comune contesto vitale, come quello del territorio, sia il risultato di visioni irriducibilmente soggettive che possono indirizzare al perseguimento di finalità non condivise disattendendo quell'accordo ideale (sistema di vincoli e regole) su cui è fondata la comunità stessa e determinando dissensi rispetto alla definizione delle possibili linee di azione ${ }^{2}$. Tale soggettività si traduce in una varietà di prospettive e punti di vista da cui traggono origine i tipici problemi di governo del territorio. Si pone, quindi, la necessità di assicurare il più ampio consenso adottando

2 Nella tematica del governo dello sviluppo del territorio è fondamentale il riferimento all'opera di Saraceno (1972). 
logiche partecipative, tenendo comunque presente che la partecipazione è condizione necessaria ma non sufficiente al raggiungimento del consenso (Saviano e Magliocca, 2003). Come evidenziato in Fig. 2, il grado di partecipazione alla vita di una comunità è espressione di un orientamento relazionale del soggetto che può variare da una logica di mera 'appartenenza', in cui gli individui prevalgono sull'insieme, a una logica 'corporativa', in cui prende forma un insieme unitario, fino all'emergere di una possibile 'coscienza' collettiva, in cui i tratti distintivi dei singoli sfumano rispetto ad una identità unitaria. Il passaggio dall'orientamento individuale a quello corporativo è attivato dal convergere verso un obiettivo comune basato su un'armonia di fini (condizioni di consonanza) che consente al sistema vitale di agire in modo sinergico e di sviluppare una visione unitaria (effetto di risonanza) (Golinelli, 2000, 2005, 2012; Barile, 2000, 2008, 2009a, 2011a) .

Fig. 2: L'emersione di una coscienza collettiva

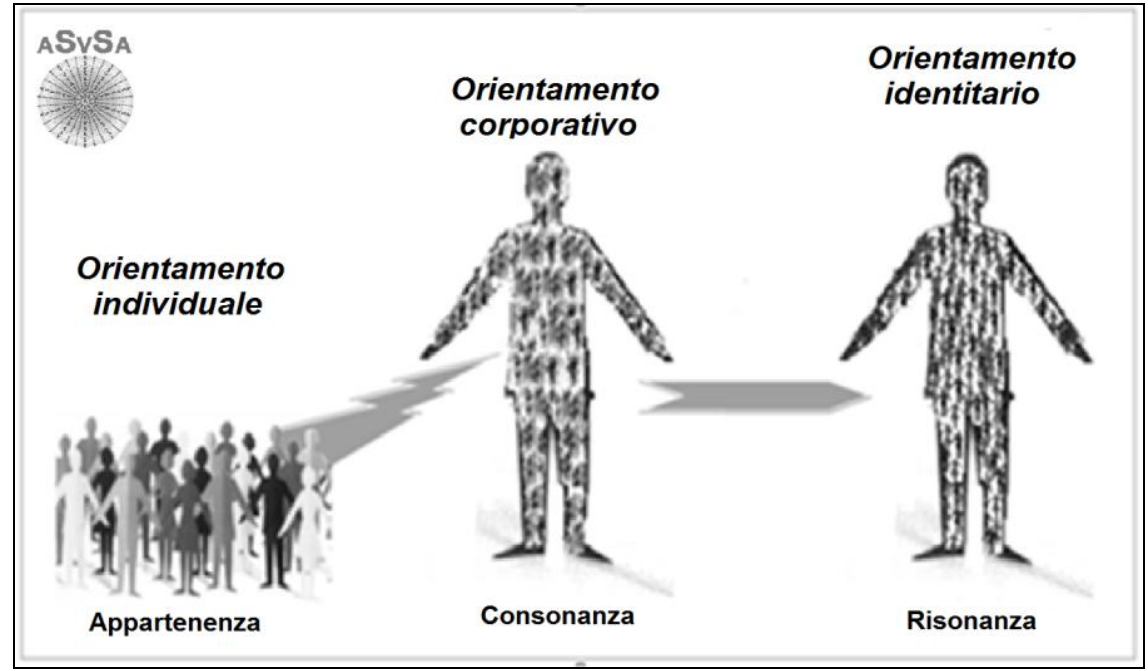

Fonte: Barile, 2012, www.asvsa.org.

Figura utilizzata con il permesso di ASvSA - Associazione per la ricerca sui Sistemi Vitali

Adottando tale prospettiva, il nostro intento è ricondurre il rapporto impresaterritorio alla dinamica di interazione tra dimensione individuale e dimensione collettiva di una comunità cercando di comprendere in che modo l'azione della componente (impresa) impatti sul sistema (territorio). In particolare, la chiave interpretativa proposta inquadra l'ampia prospettiva della sostenibilità nei rapporti

3 Una tale prospettiva rende evidente il paradosso della realtà dell'odierna società, da un lato globalizzata e sempre più interconnessa, dall'altro caratterizzata da un sempre più spinto individualismo. 
impresa-territorio in un framework teorico-concettuale reso metodologicamente coerente dal riferimento ad un comune modello di rappresentazione valido tanto per l'impresa quanto per il territorio, nonché per i soggetti che operano al loro interno. Il riferimento è alla visione di individui e organizzazioni come sistemi vitali (Golinelli, 2000; Barile, 2000), ossia come entità accomunate dall'essere sistemi aperti che ricercano condizioni di sopravvivenza nel proprio contesto vitale armonizzando le proprie finalità con quelle delle altre entità con cui si rapportano (consonanza $)^{4}$.

Sulla base di tale visione, nel seguito, si intende, quindi, sviluppare una chiave interpretativa del rapporto impresa-territorio utile al raggiungimento dell'ambizioso obiettivo di realizzare sistemi di sviluppo territoriale capaci di rispettare vincoli di efficienza locale, efficacia di contesto e sostenibilità ambientale.

\section{II territorio come sistema vitale}

La letteratura d'impresa ha manifestato particolare interesse per le problematiche dello sviluppo dei sistemi produttivi locali (Metallo, 2010), dai contributi di Marshall (1920) sui distretti industriali, transitando attraverso un processo di rifocalizzazione sul tema della localizzazione e dei distretti, operato da Becattini (1979), per indirizzare poi l'attenzione verso il concetto di rete, proposto da Rullani, 1996; Lorenzoni, 1997. Interessanti, inoltre, i modelli di corporate network di Staber (2001), e gli studi sui processi di apprendimento collettivo di Camagni (1991), Maillat (1995) e altri.

Originariamente, la definizione comune di territorio poneva l'accento sui concetti di area, spazio o regione e sulla configurazione fisica che prevalentemente possiedono, palesando il paradigma dominante di una visione strutturale che indirizzava a descriverlo oggettivamente come un insieme di risorse di dotazione (Barile e Saviano, 2008, 2011). In una proiezione più estesa, emerge la centralità dell'attività antropica e dell'incessante interazione uomo-ambiente che definisce nel tempo identità e vocazione di un territorio connotandoli di ampi e profondi significati, anche culturali, rintracciabili nelle numerose testimonianze di civiltà dell'immenso patrimonio di cui dispone l'intera umanità ${ }^{5}$.

4 La proposta metodologica è, quindi, quella dell'Approccio Sistemico Vitale $(A S V)$ alla cui letteratura si rinvia per approfondimenti. Riferimenti essenziali sono le opere di Golinelli, 2000, 2005, 2011, 2012; Barile, 2000, 2008, 2009, 2011. Una sintesi introduttiva all'approccio è proposta in Barile et al., 2012d e in http://en.wikipedia.org/wiki/Viable_systems_approach. In proposito, a parere di chi scrive, l'adozione di un comune modello di riferimento rappresenta una scelta metodologica fondamentale che, in un contesto multi soggettivo e multi stakeholder come quello del territorio, agevola l'armonizzazione delle diverse prospettive favorendo l'individuazione di linee di azione condivise.

5 Si vedano, in proposito, Golinelli, 2012; Barile, 2012; Montella, 2012; Barile et al., 2012b; Barile et al., 2012c; Barile e Saviano, 2012b. 
Con una letteratura più recente, di matrice sistemica ${ }^{6}$, il concetto di territorio si arricchisce di significati ed è interpretato come giacimento di risorse ed entità in azione per l'emersione di un sistema unitario. In questa direzione, è significativo il contributo dell'Approccio Sistemico Vitale $(A S V)$ nella concettualizzazione delle linee guida per l'adozione di una metodologia di governo per lo sviluppo del territorio. Innanzitutto, l' $(A S V)$ indirizza verso il superamento di una visione prevalentemente oggettiva e riduzionista (Barile e Saviano, 2011), che non consente di cogliere le implicazioni della varietà di prospettive e le opportunità emergenti nella dinamica sistemica (Saviano, 1999) ${ }^{7}$. Il sistema territoriale, infatti, è caratterizzato dalla presenza di componenti non solo di dotazione (naturali, artistiche, culturali, strutturali, urbanistiche, infrastrutturali, ecc.) ma anche sistemiche (imprese, organizzazioni sociali, individui, enti e istituzioni) (Barile e Golinelli, 2008). Mentre le prime appartengono 'oggettivamente' all'area geografica territoriale, le seconde godono di una precipua e autonoma capacità di generazione di valore e tendono a proiettare attese e aspettative in modo soggettivo, in ragione del perseguimento della propria sopravvivenza nel contesto territoriale di riferimento (Barile e Golinelli, 2008; Barile, 2011b). Come sistema vitale, il territorio, infatti, è caratterizzato da un Organo di Governo (OdG) composito, multisoggettivo, con un'elevata articolazione del processo decisionale, e da una Struttura Operativa (SO) tipicamente multi-dimensionale. L'OdG, in particolare, si articola in tre livelli logici (Golinelli, 2003; Barile e Golinelli, 2008):

- il Soggetto Ordinatore (S.O.), in genere il Governatore e la Giunta esecutiva, deputato all'individuazione delle linee di azione derivate da una soggettiva lettura dell'ambiente che, attraverso l'individuazione delle vocazioni, conduce all'estrazione di uno o più contesti da sottoporre all'attenzione di eventuali soggetti coordinatori;

- uno o più Soggetti Coordinatori (S.C.), capaci di sviluppare proposte nell'ambito dei contesti individuati dal S.O.;

- uno o più Soggetti Proponenti (S.P.), impegnati nella realizzazione di progetti collegati alle proposte formulate dal S.C.

6 Il riferimento è agli studi sul territorio sviluppati nell'ambito della prospettiva sistemico vitale. Cfr. Golinelli, 2003; Barile e Golinelli, 2008; Barile, 2011; Barile e Di Nauta, 2011; Barile e Saviano, 2012b; Barile et al., 2012b.

7 La prospettiva sistemica apre poi a numerose fertilizzazioni incrociate con filoni di ricerca con radici nel pensiero sistemico che, nell'ultimo decennio, hanno proposto il paradigma del servizio quale base generale dello scambio orientandosi verso una logica relazionale dove gli aspetti della condivisione e dell'interazione assumono rilievo centrale (Lusch e Vargo, 2006; Gummesson e Polese, 2009; Polese et al., 2010; Golinelli et al., 2010; Barile e Saviano, 2012a; Barile et al., 2012c; Barile et al., 2012d; Badinelli et al., 2012g; Ng et al., 2012; Aguiari e Di Nauta, 2012; Polese e Di Nauta, 2013).Il riferimento è alla Service-Dominant logic, la cui adozione nel governo del territorio è foriera di significativi contributi soprattutto verso il superamento dell'orientamento individuale a vantaggio di una prospettiva inclusiva dei diversi attori coinvolti nelle dinamiche del territorio secondo una logica di co-creazione del valore. 
L'ampia varietà di relazioni intersoggettive che caratterizza il territorio è complicata dal fatto che le tre categorie di attori, in virtù della propria dotazione di varietà informativa, ossia di informazioni, schemi interpretativi e categorie valoriali attraverso cui si esprime la loro identità sistemica da un punto di vista cognitivo (Barile, 2009a, 2011a) e, quindi, di una interpretazione soggettiva, 'vedono' diversi scenari estraendoli progressivamente da un comune ambiente. Una ulteriore complicazione deriva dal fatto che, quando il sistema 'emerge' dinamicamente dalla struttura del territorio, i confini di quest'ultima tendono a sfumare (Barile e Saviano, 2008, 2011): il solo fatto che l'OdG 'veda' un certo elemento, lo rende parte dello scenario e oggetto di possibile inclusione nel contesto estratto dall'ambiente osservato e, quindi, nel sistema vitale (Fig. 3$)^{8}$. Il passaggio da ambiente a contesto qualifica uno dei momenti più importanti nell'ottica di rappresentazione delle organizzazioni immaginata dall' $(A S V)$.

Fig. 3: Dall'ambiente al contesto al sistema vitale

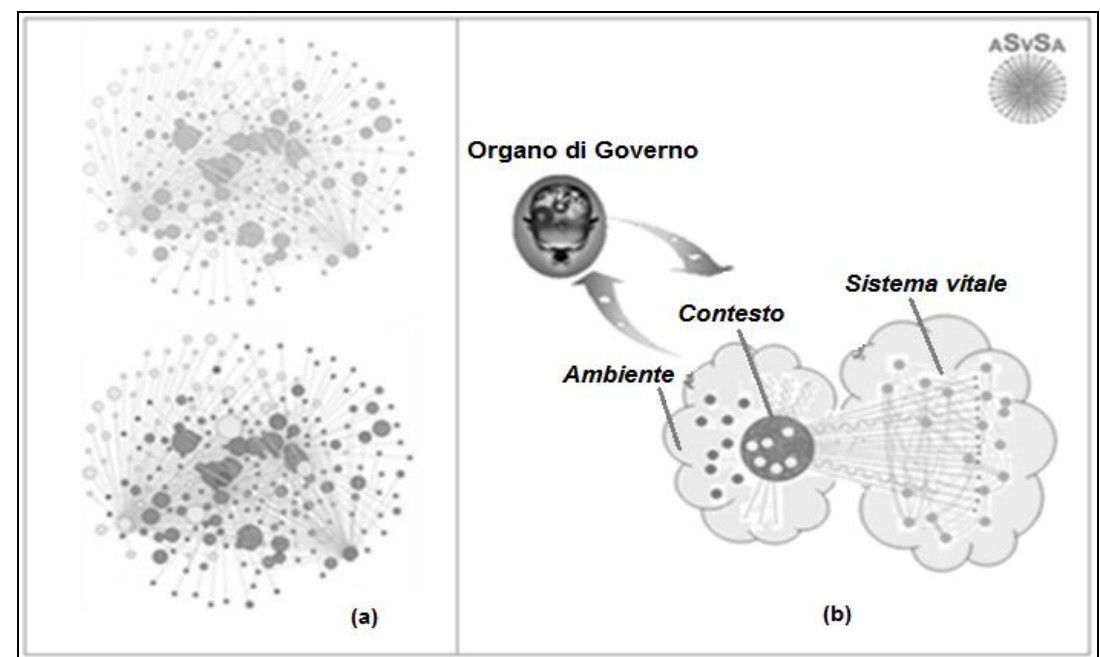

Fonte: Barile, 2010 e 2011b, p. 59, www.asvsa.org.

Figura utilizzata con il permesso di ASvSA - Associazione per la ricerca sui Sistemi Vitali

L'OdG seleziona nell'ambiente quei riferimenti (sovrasistemi) cui rivolgersi per il reperimento delle risorse, dedicandovi in via prioritaria la propria attenzione, ne interpreta le esigenze, le traduce in obiettivi e disegna la strategia per la realizzazione degli stessi, mappando le componenti necessarie e la rete di relazioni da instaurare (Polese e Minguzzi, 2009). La selezione operata dall'OdG si fonda su

8 Interessante, in proposito, la distinzione tra i concetti di territorio e di territorialità (Sassen, 2000). 
una valutazione della rilevanza dell'entità osservata come sovrasistema, che considera la criticità della risorsa detenuta e il potere di influenza esercitabile (Golinelli, 2005; Barile e Golinelli, 2008). Il sistema vitale emerge, quindi, dall'attivazione delle relazioni che danno luogo ai processi operativi territoriali (Polese, 2002). In Fig. 3 si evidenzia, quindi, come da uno stesso ambiente territoriale, diversi OdG possano estrarre diversi contesti (a) (insiemi definiti dalle diverse tonalità di grigio), delineando poi il conseguente processo di emersione del sistema vitale nella prospettiva di un OdG (b). La distinzione tra ambiente e contesto assume, quindi, particolare rilievo nel governo del territorio: se il primo è identificabile come il complesso delle risorse di diversa natura residenti nella sua proiezione geografica, il secondo è il prodotto di un'iniziativa che coinvolge e fa interagire effettivamente un sottoinsieme di tali risorse, combinandole con risorse esterne e/o con nuove risorse interne. A nostro parere, tale iniziativa deve essere concepita sulla base di una logica di sostenibilità nel tempo (opportunità e fattibilità) e di complementarità con altri contesti implementati nel territorio (compatibilità) (Barile, 2011b).

\subsection{II processo decisionale territoriale}

Riconcepito in ottica sistemica, il governo del territorio indirizza verso una visione del processo di creazione di valore non nel o del territorio, ma per il territorio, ossia per i sovrasistemi percepiti come rilevanti dall'OdG (Barile, 2011b). Se, dunque, l'OdG è di natura composita e multi-soggettiva, è chiaro che la valutazione della rilevanza dei sovrasistemi potrebbe divergere nella prospettiva dei diversi soggetti coinvolti nel processo decisionale dando luogo a dinamiche varie la cui natura e il cui esito sono generalmente riconducibili al sistema di valori guida dei diversi attori (Barile, 2009a).

Il modello di territorio sistema vitale indirizza, in sostanza, verso una rappresentazione utile a supportare decisioni di governo che migliorino le probabilità di sopravvivenza del sistema territoriale nel suo complesso anche in un'ottica di sostenibilità ${ }^{9}$. Basato su accordi di collaborazione tra le numerose entità sistemiche coinvolte nelle dinamiche del territorio, lo sviluppo deve essere cogenerato dalla pluralità di attori coinvolti, aprendo a opportunità spesso imprevedibili che l'OdG deve essere capace di intercettare. In tale contesto, come anticipato in precedenza (nota 7), l'adozione di una logica di scambio basata sul servizio, così come proposto nell'ambito della Service-Dominant logic, può agevolare il processo di co-creazione di valore indirizzando gli attori, considerati come integratori di risorse, verso una concezione di rapporto aperto e dinamico. Su un piano tecnico-operativo, invece, l'adozione dei noti strumenti della Programmazione Negoziata può efficacemente supportare l'implementazione di

9 Un'analisi delle possibili forme di relazione tra territorio, economia e potere statale che siano in grado di assicurare la sopravvivenza del complesso sociale facendo leva sulla sostenibilità è proposta in Strange (1997). 
progetti di sviluppo concertato sul territorio, regolando l'azione congiunta di una varietà di attori e portatori di interessi diversi, tra cui l'impresa assume un ruolo rilevante (Saviano e Magliocca, 2003). Ciò rende decisivo lo sforzo di sintesi compiuto dai soggetti decisori nel momento in cui selezionano, in un determinato ambiente, possibili linee d'azione per lo sviluppo convergendo verso traiettorie condivise e incrementando i livelli complessivi di consonanza (Fig. 4).

Fig. 4: Schema di sintesi del processo decisionale territoriale

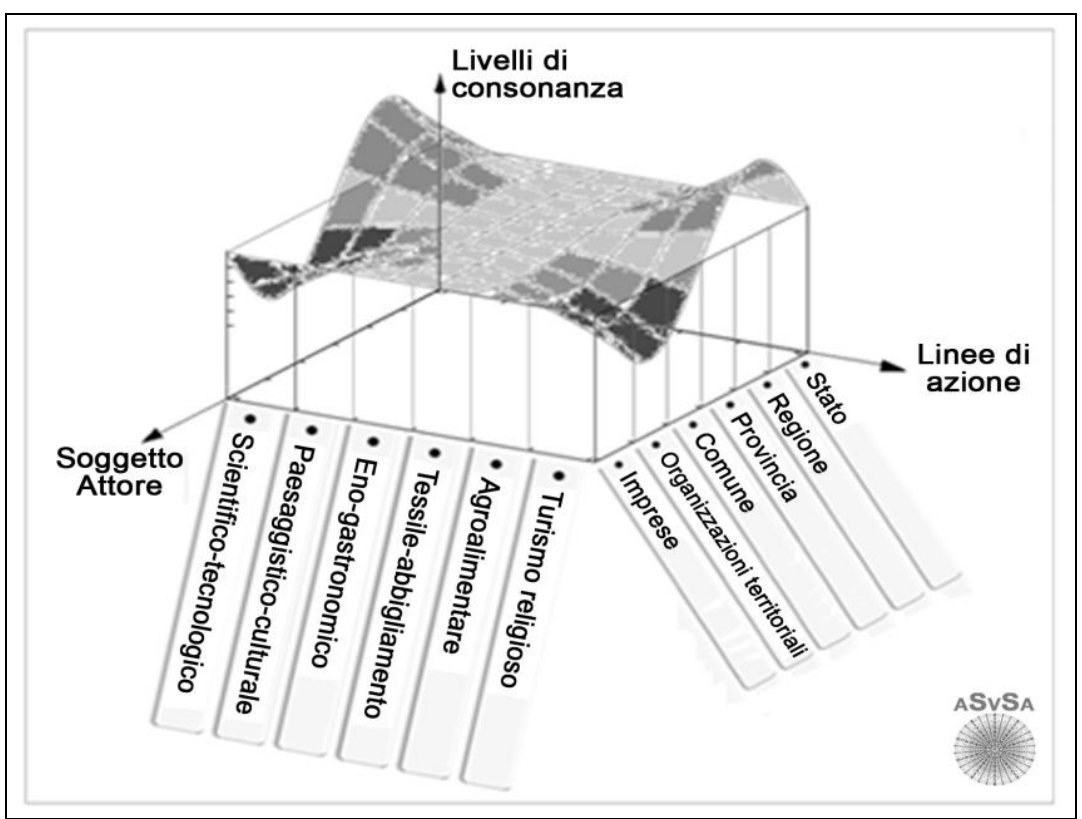

Fonte: Barile, 2010 e 2011b: 80, www.asvsa.org.

Figura utilizzata con il permesso di ASvSA - Associazione per la ricerca sui Sistemi Vitali

Come illustrato in Fig. 4, le linee di azione perseguibili vanno ricercate e sviluppate identificando le peculiari vocazioni del territorio (produttiva, turistica, storico-artistica ecc.), che rappresentano fattori di differenziazione e di attrazione sui quali basare la strategia di valorizzazione ${ }^{10}$. È su tali fattori che bisogna fondare la possibilità di instaurare rapporti di scambio al fine di reperire quelle risorse (investimenti, turisti, accordi e collaborazioni con altri territori) utili alla creazione di un vantaggio competitivo strumentale al perseguimento delle finalità di sviluppo.

10 Sulle strategie di valorizzazione in un'ottica di marketing territoriale si vedano Franch et al., 2009; Scicutella e Maizza, 2003. 
L'interpretazione proposta conduce ad una visione del territorio che consente di individuare come componenti contestuali non solo quelle fisico-naturali o di scenario, ma anche quelle che nel corso dello sviluppo naturale e umano dei luoghi e delle circostanze hanno qualificato una dotazione specifica, non solo per le 'forme', ma soprattutto per i caratteri, per la storia e per la vocazione, rendendo il rapporto impresa-territorio centrale in un'azione di governo ispirata ad una visione di sviluppo sostenibile.

\section{II rapporto impresa-territorio tra efficienza, efficacia e sostenibilità}

Le riflessioni fin qui proposte intendono indirizzare verso una visione unitaria del territorio come sistema dinamico di relazioni intersoggettive capaci di sedimentare risorse relazionali, cognitive e organizzative di tipo contestuale che possano non solo garantire l'efficienza dei processi interni all'organizzazione sviluppata, ma anche risultati efficaci nel contesto complessivo e un impatto sostenibile nell'ambiente più esteso.

$\mathrm{Si}$ propone, in sostanza, una visione del rapporto impresa-territorio come articolata realtà multi-prospettica il cui ciclo sistemico vitale disegna un'architettura complessa di livelli di osservazione e di prospettive in cui si intrecciano le molteplici dinamiche dell'economia, dell'ambiente e della società.

\subsection{Varietà, ampiezza e convergenza delle prospettive nel territorio sistema vitale}

La realtà operativa di qualsiasi sistema vitale si caratterizza per un intreccio di dinamiche che coinvolgono i portatori di una varietà di interessi generalmente difficili da conciliare. In un contesto di governo riferito a un sistema territoriale, in particolare, è necessario innanzitutto riconoscere tale varietà di prospettive, per poi renderle compatibili, se non convergenti, nell'ambito di un programma negoziato di sviluppo (Saviano e Magliocca, 2003). La negoziazione stessa è da intendersi come un processo volto a selezionare il set di priorità e attese intorno alle quali può concretamente delinearsi il consenso. Proprio ai fini del consenso, è necessario che ciascun attore attui un ampliamento di prospettiva nel valutare la fattibilità e sostenibilità, tanto economica, quanto sociale, nonché ambientale, delle linee di azione proposte adottando il punto di vista dei soggetti coinvolti e/o ad esse interessati e favorendo la convergenza verso una comune visione (Golinelli et al., 2012). Come illustrato in Fig. 5, quando il decisore osserva l'ambiente, la sua visione è soggettivamente 'limitata' dall'ampiezza della prospettiva e dai relativi schemi interpretativi. Inevitabilmente, infatti, come evidenziato, le scelte di ciascun attore sono determinate dal sistema di valori, dai paradigmi dominanti e dalle finalità perseguite in funzione delle attese dei sovrasistemi percepiti come rilevanti. La capacità di ampliare la prospettiva (Fig. 5a) consente al decisore di intercettare visioni diverse e, rifocalizzando la propria (Fig. 5b), di individuare nuovi scenari 
risolutivi e più ampi spazi di consonanza. Un simile processo, realizzato da tutti gli attori, può significativamente favorire lo sviluppo della auspicata visione condivisa di sostenibilità ${ }^{11}$.

Fig. 5: Ampliamento della prospettiva e possibili nuovi scenari risolutivi

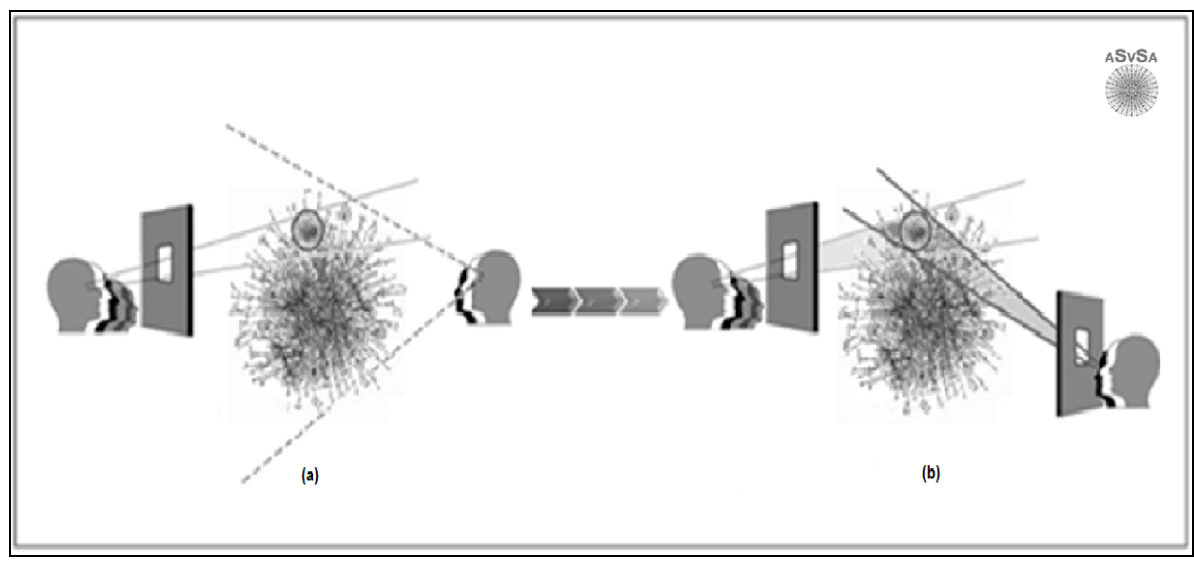

Fonte: Barile, 2012 in Golinelli et al., 2012, www.asvsa.org.

Figura utilizzata con il permesso di ASVSA - Associazione per la ricerca sui Sistemi Vitali

Centrale nell'azione di governo del territorio è, quindi, la capacità di conseguire adeguati livelli di consonanza rispetto alle linee di azione progettate (Barile, 2011b). Rispetto a tale necessità, è inoltre utile considerare che il territorio può sviluppare un funzionamento sistemico unitario tanto in virtù di dinamiche relazionali emergenti bottom-up quanto in virtù di una convergenza indotta o guidata top-down (Golinelli, $2000)^{12}$. È chiaro, tuttavia, che, data la sua caratterizzazione multi-soggettiva, è molto improbabile che si sviluppi spontaneamente una tale armonia di finalità e interessi senza che un soggetto decisore capace di indurre una visione ampia, investito della necessaria autorità e riconosciuto come tale dagli attori territoriali , ne governi dall'alto le dinamiche, indirizzando il sistema verso gli obiettivi fissati sulla base di un'ampia consonanza di contesto.

11 Il contributo della prospettiva sistemica nell'interpretazione della sostenibilità è significativo (Barile et al., 2012f). La necessità di una visione sistemica, affinché ciascuno comprenda il proprio comportamento e le relative conseguenze, è sottolineata in Clayton e Radcliffe, 1996. Una sintesi di diverse interpretazioni teoriche della prospettiva della sostenibilità, è proposta in Garriga e Melé, 2004.

12 La rilevanza del ruolo dell'OdG nello sviluppo della risonanza sistemica è sottolineata in Maggioni e Del Giudice, 2006. Un interessante studio comparativo sull'efficacia dell'attività di governo delle economie regionali è proposto in Cooke e Morgan, 1998. 
Al fine di supportare una visione ampia e inclusiva delle dinamiche del rapporto impresa-territorio, è utile, dunque, sviluppare un modello di sintesi che ponga in relazione le diverse prospettive soggettive in un quadro unitario rendendo evidente all'osservatore la necessità di innestare un circolo virtuoso. In tale contesto, l'economista d'impresa interpreta la prospettiva dell'OdG delle organizzazioni, siano esse imprese le cui azioni insistono e impattano sul territorio, siano esse istituzioni o aziende pubbliche, direttamente responsabili delle scelte di governo del territorio e dei servizi erogati alla comunità di individui, famiglie e organizzazioni. Assumere tali prospettive soggettive, tuttavia, non significa privilegiarle nel modello proposto. Tutt'altro: significa riconoscere il ruolo fondamentale svolto da tali soggetti nell'implementare un modello di sviluppo sostenibile nel territorio. Proprio per questo, nella visione proposta, è necessario che l'OdG, tanto dell'impresa quanto del territorio tutto, non trascuri di considerare il punto di vista di sovrasistemi generalmente percepiti come meno rilevanti. Il riferimento è, innanzitutto, ai cittadini, che, nella dimensione di 'insieme' della collettività, in un'ottica sistemico vitale, rappresentano la 'proprietà' del sistema territorio ossia il soggetto cui andrebbero ricondotte le attese delle scelte di governo, nonché la responsabilità ultima per le stesse, considerato il meccanismo della rappresentanza politica. Raramente, infatti, o comunque non stabilmente, la collettività riesce a sviluppare un orientamento relazionale (anche su base corporativa) adeguato a generare una forza sistemico vitale unitaria espressione di una identità collettiva. Più spesso, infatti, rimane un mero insieme caratterizzato dal prevalere di una prospettiva individuale che solo incidentalmente intercetta tendenze collettive secondo una logica di appartenenza, come precedentemente illustrato in Fig. 2. Nella varietà e sovrapposizione di ruoli che caratterizzano il territorio, è chiaro che gli stessi cittadini, sia individualmente sia attraverso entità organizzate, sono ora rappresentanti politici, ora imprenditori, ora dipendenti, ora clienti e così via, rivestendo di volta in volta ruoli diversi come portatori di interessi particolari.

Questa varietà di ruoli interpretati dagli stessi soggetti dovrebbe, di fatto, agevolare 1' 'esercizio' sistemico del cambiamento di prospettiva consentendo di approdare ad una visione unitaria delle dinamiche vitali del territorio in cui ogni singola componente (parte) sia consapevole delle proprie responsabilità nel determinare le dinamiche del sistema (tutto). Se il cambiamento di prospettiva, reso possibile dall'ampliamento dello 'sguardo', così come illustrato in Fig. 5, rappresenta la chiave del meccanismo vitale di governo del territorio, quale unica via per conseguire la ricercata consonanza, diviene allora fondamentale 'aprirsi' a una visione ampia riscoprendo il valore e la forza del 'collettivo' rispetto al singolo.

Il passaggio necessario è tanto cruciale quanto arduo poiché risente del delicato meccanismo di equilibrio tra forze di competitività e di consonanza (Golinelli et al., 2008): l'una, forza impulsiva, rappresenta la tendenza all'affermazione del sé rispetto all'altro e, esprimendo la pulsione al perseguimento delle finalità individuali, indirizza verso vantaggi di breve periodo che tendono a privilegiare $\mathrm{i}$ risultati locali (ottica micro); l'altra, forza di campo, è generata dal sentirsi parte di un contesto e dall'essere coinvolti nelle sue dinamiche aprendosi ad una visione 
ampia (ottica macro) capace di soddisfare le attese dei diversi soggetti interagenti (Golinelli, 2000, 2005; Barile, 2009b, 2011a) ${ }^{13}$. Le due forze si intrecciano e trovano composizione nella dinamica vitale del sistema attraverso stati di equilibrio dinamico. L'OdG, in sostanza, deve avere la capacità di decidere in ottica competitiva e comprendere in ottica di consonanza, stringendo e allargando opportunamente il focus tra livelli micro e macro di osservazione. Certamente, la ricorsività che caratterizza l'articolata struttura socio-economica del territorio come sistema vitale complica l'interazione tra logica di competitività e logica di consonanza rendendo arduo lo sviluppo di un'azione sinergica complessiva e critica la convergenza verso una prospettiva condivisa di sostenibilità.

\subsection{II ciclo sistemico vitale nel rapporto impresa-territorio}

Nel rapporto impresa-territorio, riletto nella prospettiva sistemico vitale, emerge una logica di reciproca necessità e di potenziale relazione sinergica, in una visione complessiva in cui l'impresa è una leva fondamentale per lo sviluppo sostenibile e il territorio è contesto e attore di quello stesso sviluppo. Una visione in cui lo sfumare dei confini tra impresa e territorio, tra imprese nel territorio e tra territori stessi diviene volano di sviluppo piuttosto che causa di perdita di controllo ${ }^{14}$. Il confine come barriera di protezione interviene, infatti, artificialmente forzando il meccanismo sistemico vitale e assicurando solo localmente e temporaneamente condizioni di accresciuta competitività ${ }^{15}$. Elevare barriere all'interazione, ostacolando il meccanismo della consonanza e svilendo la partecipazione, significa sviluppare una miopia che, pur assicurando nel breve risultati più soddisfacenti (ottica di efficienza), non porta molto lontano, rischiando di compromettere le possibilità di sopravvivenza del sistema nel più lungo periodo e nel più ampio contesto di riferimento (ottica di efficacia e di sostenibilità).

Nella visione unitaria dell'( $A S V)$, ciascuno dei criteri guida dell'azione di qualsivoglia organizzazione - efficienza, efficacia e sostenibilità - trova una precisa collocazione logica e definisce una specifica prospettiva. Il punto torna ad essere la prospettiva: il perseguimento armonico dei tre obiettivi nel governo delle dinamiche vitali del territorio e dell'impresa nel territorio non può che risultare dalla capacità di spostarsi tra la prospettiva della componente (micro) e quella del sistema complessivo (macro). Il disinteresse nel passato rispetto al 'problema' della sostenibilità, che lo ha reso ormai urgente nel presente e forse compromesso per il futuro, non è che il risultato di tale miopia, ossia dell'incapacità di ampliare e 'allungare' la prospettiva, svolgendo quel fondamentale esercizio sistemico che

13 Un'analogia efficace qualifica la consonanza 'come la corrente di un fiume' che coinvolge chi la segue orientandosi alla convergenza e alla condivisione.

14 Interessante, in proposito, la prospettiva di Zimmermann (2001) sulla relazione impresaterritorio in un contesto di globalizzazione.

15 Un'interpretazione sistemica del problema dei confini costruita sulla base di un interessante caso di studio è sviluppata in Barile et al., 2012g. 
consente di vedere oltre i confini e di considerarli come un limite, in una visione che coglie le dinamiche vitali del sistema quale entità naturalmente aperta all'interazione ${ }^{16}$. È la visione dell'intero ciclo sistemico vitale in cui si comprende l'irriducibile e profondo legame delle parti nel tutto, in cui il nesso ricorsivo impresa-territorio diviene il fulcro di uno sviluppo sinergico, che vede nella sostenibilità una naturale esigenza insita nel concetto di vitalità sistemica (Golinelli, 2000; Maizza, 2006): in sintesi, il sistema, dalla singola componente all'intero territorio, per sopravvivere, deve riuscire a conciliare obiettivi di efficienza, efficacia e sostenibilità (Saviano et al., 2010).

Il ciclo sistemico vitale rappresentato in Fig. 6 diviene così modello di riferimento attraverso il quale leggere la relazione tra impresa e territorio quali entità non separate, bensì strettamente interconnesse nella ininterrotta continuità della dinamica vitale di contesto. Nel modello del ciclo sistemico vitale, l'impresa, estraendo un contesto dall'ambiente, definisce l'ambito di legittimazione e valorizzazione del proprio ruolo e, conseguentemente, le attese di efficacia espresse dalle diverse categorie di sovrasistemi da soddisfare sulla base di strategie di contesto ispirate alla logica della consonanza. Come illustrato in Fig. 6, partendo da una visione 'positiva' della complessità come ininterrotto generarsi di nuove possibilità, l'OdG del sistema di cui si assume la prospettiva (impresa) estrae un contesto definendo l'insieme dei sovrasistemi di riferimento, ne valuta la rilevanza e identifica gli spazi di consonanza sulla base di una corretta proiezione delle relative attese (area di intersezione delle proiezioni) creando così le condizioni di efficacia del sistema vitale nel contesto. Tali attese di efficacia dovranno essere conciliate con meccanismi di funzionamento della struttura del sistema che assicurino l'efficienza necessaria ai fini di una gestione profittevole in cui l'uso delle risorse sia valorizzato.

La piena valorizzazione delle risorse, nella definizione dei meccanismi operativi della struttura, rappresenta una leva fondamentale in un approccio di gestione del sistema volto a perseguire obiettivi di efficienza ed efficacia in una ampia visione di sostenibilità. Lo sguardo dell'OdG, infatti, muove dall'osservazione dell'ambiente per poi stringere progressivamente il focus sul contesto fino alla struttura del sistema governato ed alle relative componenti (dal macro al micro), ma poi deve riallargarsi all'ambiente per monitorarne le problematiche emergenti ai fini del mantenimento nel tempo delle condizioni di vitalità (Saviano e Caputo, 2012), che si riverberano a cascata sull'impresa così come su tutti gli attori del contesto (dal micro al macro).

In tale prospettiva, la sostenibilità diviene paradigma generale di riferimento in una visione che, naturalmente collegata ai principi dell' $(A S V)$, conduce a concepirla come dimensione rilevante delle condizioni di vitalità del sistema (Barile et al., 2012f).

16 Vedere i confini come "muri" (Baccarani e Golinelli, 2012) può assicurare un senso di protezione da possibili minacce ma anche precludere importanti opportunità. 


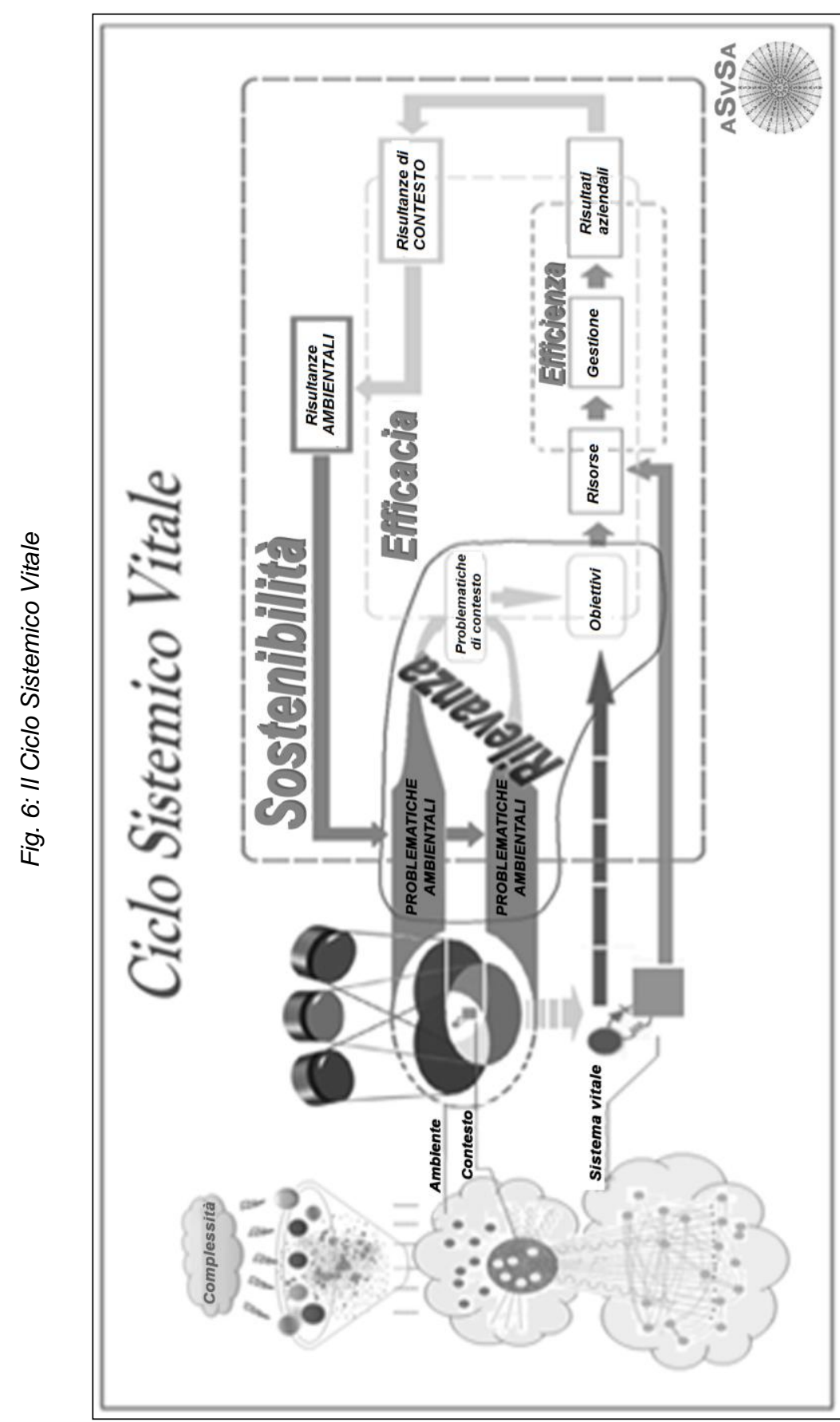

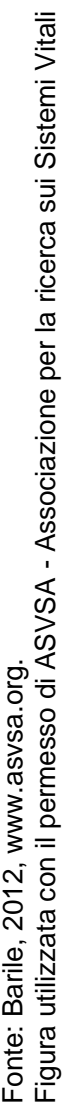


L'OdG, quindi, è responsabile della comprensione delle dinamiche di contesto e della definizione della strategia più opportuna da adottare sulla base di un 'pensiero lungo', capace di condurre a nuove configurazioni (sostenibili) del sistema. Strategia basata sulla capacità di 'anticipare' (anche immaginare) la visione di scenari futuri, da cui trarre ispirazione per le iniziative di governo e, quindi, per le strategie di azione in un'ottica che includa la sostenibilità. In questo modo, la sostenibilità può divenire driver di attivazione di relazioni di retro-causalità (Maggioni et al., 2012), ossia di una causalità inversa in cui non sono tanto gli eventi del passato a 'causare' quelli del presente quanto i traguardi che si desidera raggiungere nel futuro, espressione di un processo volitivo di governo quale azione illuminata di management capace di sollecitare, come già illustrato in Fig. 2, l'emergere di una coscienza collettiva e la convergenza verso finalità condivise (Barile, 2009a; Schillaci e Gatti, 2010).

Ne consegue una visione mutata ed evoluta del rapporto impresa-territorio quale realtà unitaria in cui in territorio, letto soggettivamente dal decisore, rappresenta la struttura ampliata da cui muove il processo di estrazione dell'insieme di sovrasistemi con cui l'organizzazione intende rapportarsi.

Ne consegue, inoltre, una visione rafforzata del concetto di esternalità (Katz e Shapiro, 1985). Nell'( $A S V)$, infatti, lo sfumare dei confini a livello sistemico rende evidente la necessità di vedere come interne al sistema (ancorché esterne alla struttura) tutte le risultanze generate dal meccanismo sistemico vitale ${ }^{17}$.

La visione proposta si presenta, dunque, densa di sviluppi e segnala al decisore la necessità di un profondo ripensamento delle logiche di azione seguite, soprattutto per quanto attiene alla visione dei 'confini' delle organizzazioni, che hanno natura strutturale e assumono rilevanza ai fini di valutazioni di efficienza dei meccanismi operativi della stessa. Una più ampia valutazione di efficacia impone l'allargamento dello sguardo alla pluralità di interlocutori rilevanti con cui il sistema si rapporta nel proprio contesto vitale. Una ancora più ampia valutazione di sostenibilità impone poi di monitorare le risultanze dell'azione del singolo quale componente dell'intero sistema ambientale nelle sue varie dimensioni naturale, economica e sociale. Occorre precisare, in proposito, che la visione di sostenibilità proposta, strettamente connessa a quella di vitalità sistemica, fa riferimento ad una concezione (quella sistemico vitale) dell'ambiente come realtà articolata e inclusiva della ampia varietà di dimensioni caratterizzanti un territorio. Mentre le dimensioni fisica, geografica, amministrativa delimitano la struttura del sistema territoriale, le dinamiche sistemiche sono il risultato di quell'intreccio (che dovrebbe essere virtuoso) che coinvolge soggetti, risorse e accadimenti nell'ambito dell'economia, dell'ambiente e della società ben al di là dei confini della struttura (Barile e Saviano, 2011).

Sulla base di quanto fin qui delineato in termini di schema generale di riferimento, si procede di seguito a proporre alcune indicazioni metodologiche utili

17 Tale aspetto apre ad interessanti linee di approfondimento che non possono essere trattate in questa sede ma che hanno rilevanti implicazioni in una visione sistemica del rapporto impresa-territorio. 
alla definizione di procedure di rilevazione, analisi e design dello sviluppo territoriale, per i cui aspetti tecnici si rinvia all'ampia letteratura di riferimento ${ }^{18}$, nel rispetto di vincoli di efficienza locale, efficacia di contesto e sostenibilità ambientale.

\section{Indicazioni metodologiche per lo sviluppo sostenibile nel e del sistema territoriale}

\subsection{II governo nel e del territorio tra competitività e consonanza}

Come anticipato nel paragrafo 2, l'azione di governo del territorio è interpretata da tre categorie di soggetti, i quali possono avere visioni diverse dell'ambiente osservato in virtù della propria dotazione di varietà. È chiaro, quindi, che la possibilità di sviluppare la consonanza necessaria ai fini dell'efficacia del complessivo processo decisionale, richiede un significativo sforzo di allineamento delle diverse varietà interagenti facendo sì che la diversità rappresenti un'opportunità di intravedere nuovi scenari piuttosto un rischio di dissensi ${ }^{19}$.

In un contesto territoriale, iniziative guidate da una logica di competitività miope in quanto basata su comportamenti opportunistici e speculativi, siano essi realizzati dalle imprese o dalle istituzioni stesse, generalmente disattendono le aspettative dei sovrasistemi rilevanti, compromettendo le probabilità di vitale sopravvivenza a lungo termine. Il punto è che, come anticipato, alcuni dei sovrasistemi di riferimento (tipicamente la collettività) non riescono ad acquisire la necessaria rilevanza nei confronti dei diversi attori in gioco e vedono così disattese le loro aspettative . Nella dinamica di rapporto con il territorio, l'impresa è non solo legittimata, ma anche tenuta ad assicurare risultati aziendali soddisfacenti; ma quando ciò accade a vantaggio dei soli soggetti direttamente interessati (per esempio, la proprietà) e a svantaggio di altri sovrasistemi percepiti come meno rilevanti (per esempio, la collettività), la competitività assicura vantaggi poco difendibili nel momento in cui i sovrasistemi della collettività (si pensi alle associazioni ambientaliste, dei consumatori, ecc.) si attivano manifestando un concreto potere di influenza. Da tale punto di vista, non pochi, invero, sono i segnali di un risveglio delle coscienze, ma siamo certamente lontani dalla realtà di una identità collettiva capace di realizzare cambiamenti rivoluzionari. Quando, invece, la forza competitiva trova composizione con la forza di consonanza, l'organizzazione riesce a sviluppare un'ampia risonanza

18 Cfr., tra gli altri, Isard, 1956; Vicari e Mangiarotti, 1999; Pencarelli e Civitarese, 2000; Valdani e Ancarani, 2000; Dezi et al., 2005; Barile e Golinelli, 2008; Barile, 2011b, 2012; Barile e Di Nauta, 2011.

19 Nella gestione della varietà in un contesto come quello del territorio, la creatività e l'innovazione sono fondamentali per lo sviluppo di un "ecosistema del valore". Cfr. Pilotti, 2011. Un'efficace combinazione creativa della varietà è utile per lo sviluppo di significative innovazioni (Barile et al.,, 2012a; Barile et al., 2012e). 
(generazione di valore) nel contesto migliorando le probabilità di vitale sopravvivenza per una più ampia varietà di soggetti interessati, diversificando il rischio e rafforzando così le basi della stessa competitività.

Appare chiaro, quindi, che la chiave dell'equilibrio tra competitività e consonanza è nel favorire una visione non dicotomica delle due forze, cogliendo l'ininterrotto legame della parte nel tutto, della componente nel sistema, in una visione unitaria dell'intero ciclo sistemico vitale.

Il processo decisionale nel e del sistema vitale territorio richiede, quindi, una forte capacità di governance indirizzata alla valorizzazione delle potenzialità e delle vocazioni riferibili all'area con ricadute positive in termini di opportunità, benessere, qualità della vita, ecc. per l'insieme degli attori interessati. Questi ultimi, come illustrato in precedenza, in virtù della risorsa detenuta e del potere di influenza esercitabile, esprimeranno precise attese di risultato nei confronti del sistema osservato (sia esso l'intero territorio o la singola componente impresa) definendo i relativi obiettivi di efficacia. Riguardo alla più ampia ottica di sostenibilità, quale tutela di una attesa sovrasistemica 'non attuale' in quanto relativa a generazioni future, nella misura in cui non si sviluppa una soggettività collettiva unitaria che ne recepisca le istanze e sia ad essa concretamente interessata, tale azione di pressione e controllo sovrasistemico non si realizza. Anche se idealmente riconoscibile come la finalità più ampiamente condivisibile da tutti gli attori coinvolti nelle dinamiche del territorio nelle molteplici prospettive dell'economia, dell'ambiente e della società, non vi è una categoria specifica di soggetti 'interessati' che siano non solo rilevanti ma anche concretamente attivi nel manifestare un'attesa sovrasistemica. Per quanto naturalmente tutti possano dichiarare un'attenzione alla sostenibilità, nel sistema delle priorità finisce per essere di fatto collocata a valle degli obiettivi di efficienza e di efficacia relativi ad attività di più immediato interesse. L'attenzione è inevitabilmente concentrata su problematiche relative a bisogni/interessi che appaiono di più 'urgente' soddisfazione ${ }^{20}$.

Appare chiaro, quindi, come la sostenibilità sia un obiettivo spesso sacrificato nel sistema delle urgenze di un'impresa, così come di un territorio/paese, quando prevale una concezione dicotomica del rapporto tra competitività e consonanza. Di fatto, tuttavia, come sottolineato in precedenza, ciò è solo il frutto di una visione miope associata a uno spinto individualismo che ostacola lo sviluppo o il risveglio di una coscienza collettiva sensibile alle vere priorità tanto dell'uomo comune quanto dell'operatore economico investiti oggi da una crescente incertezza per quanto attiene al futuro dell'economia, dell'ambiente e della società. In sostanza, nel gioco ricorsivo del rapporto impresa-territorio-ambiente, la sfida è l'armonizzazione dei

20 Si pensi, per esempio, a quanto accade nel sistema sanitario per la prevenzione: sono ormai insostenibili tutti gli interventi assistenziali (cura e riabilitazione) che si rendono necessari a causa dell'inefficace mantenimento dello stato di salute della collettività che dovrebbe innanzitutto far leva sulla prevenzione migliorando le condizioni di sostenibilità del sistema con ricadute positive per la maggior parte dei soggetti interessati (Saviano, 2012). 
tre obiettivi di efficienza, efficacia e sostenibilità nella consapevolezza del fatto che, se il territorio è un "giacimento di vitalità per l'impresa", allora l'impresa deve essere motore di vitalità per il territorio in una prospettiva generale e condivisa di sostenibilità.

\subsection{Un modello di sintesi per le decisioni di governo del territorio: I'Ipercubo (ASV)}

Al fine di fornire un quadro di sintesi, utile nel processo decisionale di governo tanto alla singola componente impresa quanto all'intero sistema territoriale, che riassuma l'insieme delle dimensioni considerate nel presente lavoro e di quelle alla base del management sistemico vitale (Barile, 2009a), si propone di seguito il modello dell'Ipercubo $(A S V)$. Come rappresentato in Fig. 7, sulla base dello schema interpretativo proposto, è possibile qualificare i contesti problematici delle decisioni di governo del territorio, tenendo conto:

- del livello di responsabilità del soggetto decisore (politico, dirigenziale, amministrativo e operativo);

- della tipologia di decisione (strategica, direzionale, tattica e operativa);

- del livello di problematicità percepito dal decisore (caos, complessità, complicazione, certezza);

- delle dimensioni della varietà informativa rilevanti (unità informative, schemi interpretativi generali e di sintesi e categorie valoriali).

Per comprendere la logica di fondo del modello, si consideri, a titolo di esempio, il contesto problematico della decisione, attualmente in discussione nel nostro Paese nell'ambito della strategia di spending review, di accorpamento di strutture amministrative locali. Si tratta di una decisione che investe responsabilità a livello sia politico sia amministrativo (prima dimensione) e certamente di natura strategica (seconda dimensione). Orbene, mentre tale decisione può risultare non particolarmente complicata nella prospettiva dell'OdG politico statale, essa potrà apparire ben più complessa nella prospettiva delle diverse amministrazioni locali (terza dimensione). L'introduzione della quarta dimensione della 'varietà informativa' (Barile, 2009b) offre un riferimento fondamentale per il decisore, evidenziando le possibili difficoltà attuative derivanti da 'distanze cognitive' tra le varietà dei soggetti coinvolti nel processo di ristrutturazione dovute a visioni e posizioni diverse degli attori investiti da tale intervento di razionalizzazione (Barile et al., 2012a). Difficoltà certamente esasperate da una comune volontà di ostacolare un cambiamento che indubbiamente si tradurrebbe in 'perdite' di posizioni, che è espressione di un atteggiamento individualista (prospettiva della competitività); ma difficoltà anche derivanti dalla possibile distanza tra usi, costumi e consuetudini diversi, dominanti nei territori da accorpare (prospettiva della consonanza). Se, come è agevole ipotizzare, nel tempo ogni territorio matura una sua storia e sviluppa una identità e una vocazione, non è detto che la prossimità geografica (aspetto strutturale), sia pure accompagnata da una più o meno omogenea morfologia del territorio, sia sufficiente a rendere compatibili i due sistemi (o sub-sistemi) 
territoriali. Si tratta, dunque, di valutare le condizioni effettive di consonanza, adottando una visione più ampia, che consideri usi, tradizioni, consuetudini e, soprattutto, i valori di riferimento; in sintesi, categorie valoriali, che potrebbero divergere in comunità cognitivamente 'distanti' anche se geograficamente vicine, imponendo l'implementazione di un'impegnativa strategia di consonanza dall'esito incerto. La decisione investe, quindi, le dimensioni più critiche della varietà informativa e la valutazione e il monitoraggio delle condizioni di consonanza assumono rilievo centrale nel rendere fattibile e sostenibile la scelta.

Fig. 7: L'Ipercubo (ASv) per il governo del territorio

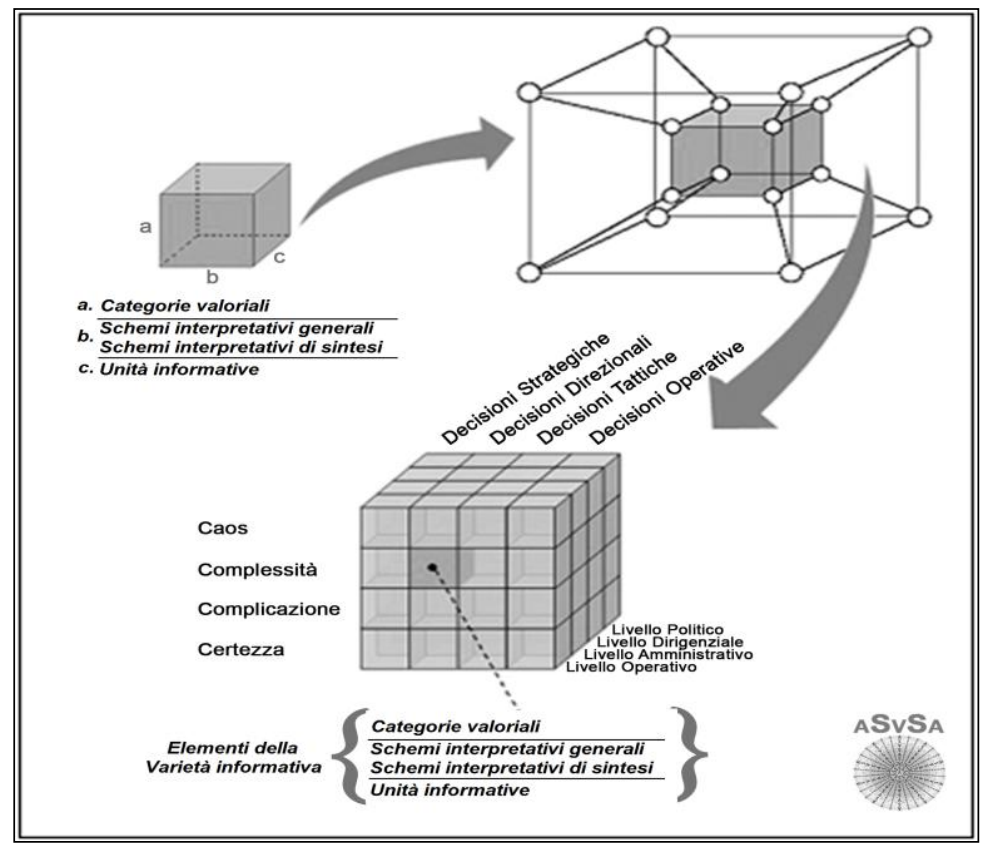

Fonte: Barile, 2012, www.asvsa.org.

Figura utilizzata con il permesso di ASVSA - Associazione per la ricerca sui Sistemi Vitali

La sostenibilità rappresenta, quindi, nell'( $A S V)$, un 'termometro' della vitalità inter-sistemica, declinabile a tutti $\mathrm{i}$ livelli relazionali ${ }^{21}$, in grado di segnalare il superamento dei limiti di 'elasticità' del rapporto con i sovrasistemi detentori delle

21 La visione relazionale agevola significativamente la ricostruzione delle formazioni sistemiche attive sul territorio. Si veda, in proposito, il lavoro di Dicken e Malmberg (2001). 
risorse e la necessità di interventi di trasformazione o di ristrutturazione, espressione di una fondamentale flessibilità, adattiva o innovativa, del sistema (Golinelli, 2011).

Gli schemi generali proposti in Fig. 6 e in Fig. 7 si collocano, dunque, nella dotazione del decisore come meta-modelli a supporto del processo decisionale che possono essere adottati, congiuntamente ai modelli manageriali consolidati, tanto dall'attore istituzionale che vede il territorio nel suo complesso come sistema vitale, quanto dall'attore imprenditoriale che vede nel territorio un fertile contesto di azione, 'giacimento di vitalità', dal quale trarre e al quale offrire opportunità di sviluppo in un intreccio armonico di finalità e in un'ottica condivisa di sostenibilità.

\section{Note conclusive}

La possibilità di formalizzare procedure di rilevazione, analisi e design, utili a favorire lo sviluppo territoriale nel rispetto di vincoli di efficienza locale, efficacia di contesto e di sostenibilità ambientale è certo un obiettivo non facilmente raggiungibile. Nel proporre schemi interpretativi di sintesi delle fondamentali dinamiche del legame ricorsivo tra impresa, attori del contesto territoriale e dell'ambiente più generale di riferimento, il contributo offre spunti di riflessione sulla delicata relazione impresa-territorio ponendola in una luce nuova densa di significati e di stimoli.

L'imperativo della sostenibilità impone ai diversi attori del territorio, in primis l'individuo e l'impresa, un ripensamento profondo delle logiche di azione sulla base di una nuova consapevolezza dell'irriducibile nesso che lega ogni entità in un'unica grande rete vitale (Capra, 1997). Solo sulla base di tale consapevolezza, ogni singola entità saprà sviluppare capacità di regolazione necessarie al fine di preservare le condizioni di equilibrio dell'intero sistema. Un simile cambiamento, tuttavia, non è di agevole attuazione in quanto deve maturare a livello culturale modificando il sistema di valori e orientando verso una equilibrata composizione delle forze di competitività e consonanza nelle dinamiche dei rapporti tra le diverse entità coinvolte nel ciclo sistemico vitale.

La visione proposta, in sintesi, individua una chiave interpretativa in grado di supportare i decisori nel governo delle articolate dinamiche del ciclo sistemico vitale territoriale indirizzandoli verso un'armonizzazione degli obiettivi di efficienza, efficacia e sostenibilità ricomposti nella più ampia e generale visione di vitalità sistemica.

\section{Bibliografia}

AGUIARI R., DI NAUTA P. (2012), “Governing Business Dynamics in Complex Contexts", Mercati e Competitività, n. 1, pp. 39-59.

BACCARANI C., GOLINELLI G.M. (2012), "Gli studi di management tra muri e ponti”, Sinergie, n. 87, pp. 7-8. 
BADINELLI R., BARILE S., NG IRENE C.L., POLESE F., SAVIANO M., DI NAUTA P. (2012), "Viable Service Systems and Decision Making in Service Management", Journal of Service Management, vol. 23, n. 4, pp. 498-526.

BARILE S. (2000), Contributi sul pensiero sistemico in economia d'impresa, ARNIA, Salerno.

BARILE S. (a cura di) (2008), L'impresa come sistema. Contributi sull'Approccio Sistemico Vitale $(A S V)$, Giappichelli, Torino.

BARILE S. (2009a), Management sistemico vitale, Giappichelli, Torino.

BARILE S. (2009b), "Verso la qualificazione del concetto di complessità sistemica", Sinergie, n. 79, pp. 47-76.

BARILE S. (2011a), Management sistemico vitale. Scelte e decisioni in ambito complesso, International Printing, Distribuzione Kappa, Roma.

BARILE S. (2011b), “L'approccio sistemico vitale per lo sviluppo del territorio”, Sinergie, n. 84, pp. 47-87.

BARILE S. (2012), "Verso una novata ipotesi di rappresentazione del concetto di bene culturale", in Golinelli G.M. (a cura di), Patrimonio culturale e creazione di valore. Verso nuovi percorsi, Cedam, Padova, pp. 71-96.

BARILE S., GOLINELLI C.M. (2008), "Modalità e limiti dell'azione di governo del territorio in ottica sistemica", in Barile S. (a cura di), L'impresa come sistema. Contributi sull'Approccio Sistemico Vitale (ASV), Giappichelli, Torino, pp. 243-268.

BARILE S., DI NAUTA P. (2011), "Viable Systems Approach for territory development", in Various Authors, Contributions to theoretical and practical advances in management - A Viable Systems Approach (VSA), International Printing, Avellino, pp. 199-243.

BARILE S., FRANCO G., NOTA G., SAVIANO M. (2012a), "Structure and Dynamics of a "T-Shaped" Knowledge. From Individuals to Cooperating Communities of Practice", Service Science, Informs vol. 4, n. 2, pp. 161-180.

BARILE S., GOLINELLI G. M., MONTELLA M., SAVIANO M. (2012b), “A systems view of cultural heritage. The case of landscape", in Morvillo A. (ed.), Advances in Tourism Studies. In memory of Clara S. Petrillo, Collana "Services and Competitiveness", McGraw-Hill Education, New York, pp. 361-379.

BARILE S., MONTELLA M., SAVIANO M. (2012c), "A Service-Based Systems View of Cultural Heritage", Journal of Business Market Management, vol. 5, n. 2, pp. 106136.

BARILE S., PELS J., POLESE F., SAVIANO M. (2012d), "An Introduction to the Viable Systems Approach and its Contribution to Marketing", Journal of Business Market Management, vol. 5, n. 2, pp. 54-78.

BARILE S., POLESE F., SAVIANO M. (2012e), Immaginare l'innovazione, Giappichelli, Torino.

BARILE S., SAVIANO M. (2008), "Le basi del pensiero sistemico: la dicotomia strutturasistema", in Barile S. (a cura di), L'impresa come sistema, Giappichelli, Torino.

BARILE S., CALABRESE M. (2009), "The value of enterprise in optical Vital Systemic", The 11th International Conference of Society for Global Business and Economic Development (SGBED), 27-30 May, Bratislava, Slovak Republic.

BARILE S., SAVIANO M. (2010), "A new perspective of systems complexity in service science", Impresa, Ambiente, Management, vol. 4, n. 3, pp. 375-414.

BARILE S., SAVIANO M. (2011), "Foundations of systems thinking: the structure-system paradigm", in Various Authors, Contributions to theoretical and practical advances in management. A Viable Systems Approach (VSA), International Printing, Avellino, pp. 1-26. 
BARILE S., SAVIANO M. (2012a), "Oltre la partnership: un cambiamento di prospettiva”, in Esposito De Falco S., Gatti C. (a cura di), La consonanza nel governo dell'impresa. Profili teorici e applicazioni, Franco Angeli, Milano, pp. 56-78.

BARILE S., SAVIANO M. (2012b), "Dalla gestione dei beni culturali al governo del sistema dei beni culturali", in Golinelli G.M. (a cura di), Patrimonio culturale e creazione di valore. Verso nuovi percorsi, Cedam, Padova, pp. 97-148.

BARILE S., SAVIANO M., IANDOLO F. (2012f), "L'innovazione tra creatività e sostenibilità", in Barile S., Polese F., Saviano M., Immaginare l'innovazione, Giappichelli, Torino.

BARILE S., SAVIANO M., POLESE F., DI NAUTA P. (2012g), "Reflections on Service Systems Boundaries: A Viable Systems Perspective. The case of the London Borough of Sutton", European Management Journal, vol. 30, n. 5, pp. 451-465.

BECCATTINI G. (1979), "Dal settore industriale al distretto industriale. Alla ricerca dell'unità di indagine dell'economia industriale", Rivista di Economia e Politica Industriale, n.1, pp. 5-25. Ora in Becattini G. (2000), Il distretto industriale, Rosenberg \& Sellier, Torino.

CAMAGNI R. (1991), "Technological change, uncertainty and innovation networks: towards a dynamic theory of economic space", in Camagni R. (ed.), Innovation networks: spatial perspectives, Bellhaven-Pinter, London.

CAPRA F. (1997), The Web of Life, Flamingo, London.

COOKE P., MORGAN K. (1998), The Associational Economy. Firms, Regions, and Innovation, Oxford University Press, Oxford.

CLAYTON A.M.H., RADCLIFFE N.J. (1996), Sustainability: A Systems Approach, Earthscan Publication Limited, London.

DEZI L., GILARDONI A., MIGLIETTA A., TESTA F. (2005), Economia e management delle imprese di pubblica utilità, Cedam, Padova.

DICKEN P., MALMBERG A. (2001), "Firms in Territories: A Relational Perspective", Economic Geography, October, vol. 77, n. 4, pp. 345-363.

FRANCH M., MARTINI U., BARBERA M.S. (2009), "Eventi e marketing territoriale: gli aspetti turistici di valorizzazione dei territori", in Paiola M., Grandinetti R. (a cura di), Città in festival. Nuove esperienze di marketing territoriale, Franco Angeli, Milano, pp. 55-76.

GARRIGA E., MELÉ D. (2004), “Corporate Social Responsibility, Theories: Mapping the Territory", Journal of Business Ethics, vol. 53, n. 1-2, pp. 51-71.

GOLINELLI C.M. (2003), Il territorio sistema vitale, Giappichelli, Torino.

GOLINELLI G.M. (2000), L'approccio sistemico al governo dell'impresa. La dinamica evolutiva del sistema impresa tra economia e finanza, vol. II, Cedam, Padova.

GOLINELLI G.M. (2005), L'approccio sistemico al governo dell'impresa. L'impresa sistema vitale, II ed., Cedam, Padova.

GOLINELLI G.M. (2011), L'approccio sistemico vitale (ASV) al governo dell'impresa. Verso la scientificazione dell'azione di governo, vol. II, II ed., Cedam, Padova.

GOLINELLI G.M. (a cura di) (2012), Patrimonio culturale e creazione di valore. Verso nuovi percorsi, Cedam, Padova.

GOLINELLI G.M. (2012), L'approccio sistemico (ASV) al governo dell'impresa. L'impresa sistema vitale, vol. I, Cedam, Padova.

GOLINELLI G.M., PROIETTI L., VAGNANI G. (2008), "L'azione di governo tra competitività e consonanza", in Golinelli G.M., L'Approccio Sistemico Vitale (ASV) al governo dell'impresa, Cedam, Padova, pp. 3-50. 
GOLINELLI G.M., BARILE S., SAVIANO M., POLESE F. (2012), "Perspective Shifts in Marketing: Toward a Paradigm Change?", Service Science, vol. 4, n. 2, pp. 121-134.

GOLINELLI G.M., BARILE S., SPOHRER J., BASSANO C. (2010), "The Evolving Dynamics of Service Co-creation in a Viable Systems Perspective", The $13^{\text {th }}$ ToulonVerona Conference, Coimbra - Portugal, September 2-4.

GUMMESSON E., POLESE F. (2009), "B2B Is Not an Island", Journal of Business \& Industrial Marketing, vol. 24, n. 5, pp. 337-350.

ISARD W. (1956), Localizzazione e spazio economico, IEC, Milano.

KATZ M.L., SHAPIRO C. (1985), "Network Externalities, Competition, and Compatibility", The American Economic Review, vol. 75, n. 3, pp. 424-440.

LORENZONI G. (1997), "Le reti interimpresa come forma organizzativa distinta", in Lomi A., L'analisi relazionale delle organizzazioni, Il Mulino, Bologna.

LUSCH R.F., VARGO S.L. (eds., 2006), The Service-Dominant Logic of Marketing - Dialog, Debate, and Directions, ME Sharpe, Armonk.

MAGGIONI V., DEL GIUDICE M. (2006), "Relazioni sistemiche tra imprenditorialità interna e gemmazione d'impresa: una ricerca empirica sulla natura cognitiva delle nuove imprese", Sinergie, n. 71, pp. 171-197.

MAGGIONI V., BARILE S., CALABRESE M., IANDOLO F. (2012), "Supercausalità, coscienza e decisioni manageriali", XXXV Convegno annuale AIDEA - Management senza confini. Gli studi di management: tradizione e paradigmi emergenti, Salerno, 4 5 Ottobre.

MAILLAT D. (1995), "Territorial dynamics, innovative milieu and regional policy", Entrepreneurship and Regional Development, vol. 7, n. 2, pp. 157-165.

MAIZZA A. (2006), La gestione d'impresa tra vitalità e rischio di crisi, Cacucci, Bari.

MARSHALL A. (1920), Principles of Economics, Macmillan, London.

METALLO G. (2010), "Indirizzi di saluto e apertura dei lavori”, Sinergie, n. 84, pp. 1-2.

MIGLIETTA A. (2008), "Cultura, conoscenza e impresa per la valorizzazione del territorio: il caso Piemonte”, Sinergie, n. 76, pp. 119-124.

MONTELLA M. (2012), "Valore culturale", in Golinelli G.M. (a cura di), Patrimonio culturale e creazione di valore. Verso nuovi percorsi, Cedam, Padova, pp. 3-70.

NG I.C.L., BADINELLI R., POLESE F., LOBLER H., HALLIDAY S., DI NAUTA P. (2012), "S-D Logic Research Directions and Opportunities: The Perspective of Systems, Complexity and Engineering", Marketing Theory, pp. 213-217.

PAINTER J. (2006), "Territory-network", Association of American Geographers Annual Meeting, Chicago, USA, 7-11 March.

PENCARELLI T., CIVITARESE C. (2000), “Appunti per un nuovo modello di governo dei distretti turistici”, Prisma, marzo, pp. 1-20.

PILOTTI L. (2011), Creatività, innovazione e territorio: ecosistemi del valore per la competizione globale, Il Mulino, Bologna.

POLESE F. (2002), "L'approccio sistemico vitale per l'analisi del territorio: Il caso del Parco Nazionale del Vesuvio", Esperienze d'impresa, n. 2/02, pp. 121-139.

POLESE F., DI NAUTA P. (2013), "A Viable Systems Approach to Relationship Management in S-D Logic and Service Science", Business Administration Review, Schäffer-Poeschel, vol. 73, n. 2 (in corso di stampa).

POLESE F., CARRUBBO L., RUSSO G. (2010), "Managing business relationships. Between Service Culture and Viable Systems Approach”, Esperienze d'impresa, pp. 135-158. 
POLESE F., MINGUZZI A. (2009), "Networking approaches for sustainable destination management: an Italian case study", in Kozac M., Andreu L., Knoth J. (a cura di), Advances in Tourism Marketing. Managing Networks, Routledge, London, pp. 113124.

RULLANI E. (1996), "Reti globali e reti metropolitane per l'economia del Nordest", in Banco Ambrosiano Veneto (a cura di), Nord-Est: fattori di competitività, Il Mulino, Bologna.

SARACENO P. (1972), Il governo delle aziende, Libreria Universitaria, Venezia.

SASSEN S. (2000), "Territory and Territoriality", Global Economy International Sociology, June, n. 15, pp. 372-393.

SAVIANO M. (1999), "La strategia come scelta emergente dal dinamismo ambientale. Una rilettura del tema alla luce della visione sistemica", Esperienze d'Impresa, n. 1, pp. 107-128.

SAVIANO M. (2012), Condizioni di efficacia relazionale e di performance nelle aziende sanitarie, Giappichelli, Torino.

SAVIANO M., BASSANO C., CALABRESE M., (2010), "A VSA-SS Approach to Healthcare Service Systems. The Triple Target of Efficiency, Effectiveness and Sustainability", Service Science, vol. 2 n. 2, pp. 41-61.

SAVIANO M., CAPUTO F. (2012), "Le scelte manageriali tra sistemi, conoscenza e vitalità", in XXXV Convegno annuale AIDEA - Management senza confini. Gli studi di management: tradizione e paradigmi emergenti, Salerno, 4-5 Ottobre.

SAVIANO M., IORIO G. (2010), "How far from participatory governance? A survey on edemocracy in Italian municipalities", PRAGYAA Journal of Management, vol. I, n. II, pp. 1-18.

SAVIANO M., MAGLIOCCA P. (2003), "Programmazione negoziata e governo del territorio. Modelli, tecniche e strumenti", Esperienze d'Impresa, n. 9, Serie Speciale 2, pp. 163-182.

SCHILLACI C.E., GATTI C. (2010), "E pluribus unum: intenzionalità collettiva e governo dei sistemi territoriali”, Sinergie, n. 84, pp. 21-45.

SCICUTELLA M., MAIZZA A. (2003), Elementi di marketing. I fondamenti della disciplina. Casi di marketing territoriale, I Liberrimi, Lecce.

STABER U. (2001), "The Structure of Networks in Industrial Districts", International Journal of Urban and Regional Research, vol. 25, n. 3, pp. 537-552.

STRANGE S. (1997), "Territory, State, Authority and Economy: A New Realist Ontology of Global Political Economy", in Cox R.W. (ed.), The New Realism: Perspectives on Multilateralism and World Order, United Nation University Press, Tokyo, pp. 3-19.

VALDANI E., ANCARANI F. (2000), Strategie di marketing del territorio. Generare valore per le imprese e i territori nell'economia della conoscenza, Egea, Milano.

VICARI S., MANGIAROTTI D. (1999), "Il marketing delle grandi città", Sinergie, n. 49, pp. 85-99.

ZIMMERMANN J.B. (2001), "The firm/territory relationships in the globalisation: towards a new rationale", European Journal of Economic and Social Systems, vol. 15, n. 1, pp. 57-75.

\section{Sitografia}

http://en.wikipedia.org/wiki/Viable_systems_approach http://www.asvsa.org 
